\title{
Thermomechanical Analysis of Four-lobe Rotors in a Roots-type Multi-Recompression Heater
}

\author{
HONGLU WANG* and JAMES D. FELSKE ${ }^{\dagger}$ \\ Mechanical and Aerospace Engineering Department, State University of New York \\ at Buffalo, Buffalo, NY 14260-440, USA
}

\begin{abstract}
The Multi-Recompression Heater (MRH) is a mechanical device which compressively heats a gas stream to very high temperatures. Potential applications are extensive. The present study analyzes the thermal, stress, and displacement fields experienced by four-lobe impellers operating under a variety of typical conditions. The ABAQUS finite element code was used for the computations. Various transient convective boundary conditions were simulated in the thermal-stress/ displacement analyses. A variety of pressure distributions and rotational speeds were considered in determining the mechanical stresses and displacements.
\end{abstract}

Keywords: Thermal stress; High temperature; Multi-Recompression Heater; Rotary-lobe; Finite element method; Stress analysis

\section{INTRODUCTION}

In principle, the Multi-Recompression Heater (MRH) heats a gas stream through a series of compressions and nearly free Joule expansions. For producing high temperature gas, it has significant advantages when compared with conventional compressors or plasma arcs. The Multi-Recompression Heater can provide an uncontaminated, quasi-steady flow of gases uniformly heated to up to $5000 \mathrm{~K}$. The potential applications of this device are extensive for the largevolume chemical and petrochemical industries as well as for hypersonic wind tunnel research. It offers unique opportunities to explore a variety of pyrolytic chemical processes from cost-effective chemical syntheses to environmentally benign destruction of hazardous and nonhazardous waste materials. The basic thermodynamic concept and method of operation of the Multi-Recompression Heater are well

\footnotetext{
Received 21 June 2000; in final form 4 August 2000.

* Corresponding author. Tel.: 716-645-2593, Fax: 716-645-3875. E-mail: hw4@eng.buffalo.edu

${ }^{\dagger}$ E-mail: felske@acsu.buffalo.edu
}

known, but challenges remain for design optimization and fabrication of a reliable prototype.

The basic principles for the operation of the device can be illustrated using Figure 1-1 (Weatherston, 1969; Dubrin \& Harri, 1980). Consider a piston and cylinder combination which is attached to a large adiabatic reservoir. In the first illustration of Figure 1-1, the top of the cylinder at the entrance to the reservoir is closed by a removable cover. In the second illustration, the piston is withdrawn to the bottom dead center position with no work required in the process and no energy loss from the reservoir. Then the cover over the cylinder is removed, increasing the volume of the reservoir. The reservoir gas expands into the displaced cylinder volume in the third illustration. During this process, no work is done and no energy is lost from the reservoir; it is a free Joule expansion. An amount of work equal to the product of the reservoir pressure and the displaced cylinder volume must be put into the system as the piston is pushed again to the entrance of the reservoir, as shown in the fourth illustration of Figure 1-1.

This cycle can be repeated. For each expansion and recompression, additional energy is added to the system. Hence, by multi-recompression, very high temperatures can be generated. A practical heater device capable of multi-recompression will necessarily have a rotary (rather than reciprocating) mechanical configuration.

A Multi-Recompression Heater was designed and built in 1978-1979 by Lawrence Livermore National Laboratory (LLNL) in conjunction with the Calspan Corporation. It was the only such device ever built. At that time, LLNL was exploring laser isotope separation technologies for the recovery of trace amounts of fissionable uranium in the 'tails' of other conventional uranium-purification processes. The Multi-Recompression Heater was to have been used for achieving a steady flow of uranium vapor atoms. The design parameters are given in Russo (1997).

The Multi-Recompression Heater was built and tested at LLNL from May, 1978 to December, 1978. It was successfully operated at $6700 \mathrm{RPM}$ under no-load 

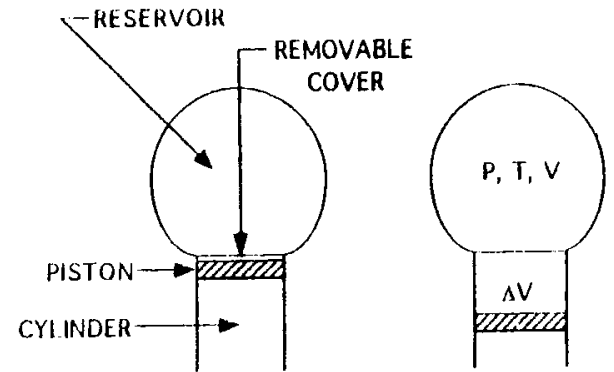

COVER

PISTON
ON

TDC

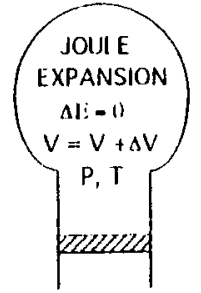

OFF

BDC
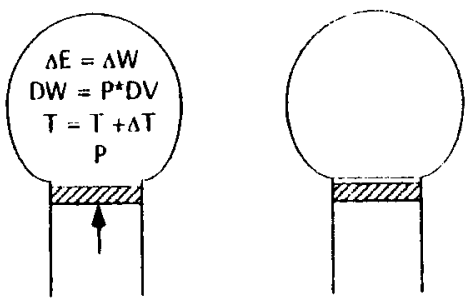

OFF

TDC
ON

TDC

FIGURE 1-1

conditions. On December 12, 1978, the plan was to accelerate the machine from 6000 RPM to 7500 RPM. However, at a rotor speed of about $7220 \mathrm{RPM}$ there was an instantaneous and catastrophic failure of the device. Failure inspections showed that the immediate cause appeared to be severe contact between the meshing lobes of the rotors.

As illustrated in Figure 1-2, contact was made at two locations on each lobe. These contact locations lay near the junction of the circular arc and involute profiles on each lobe. Failure analyses suggested a number of possibilities. Included among these were problems arising from the torsional twist of the impellers and the thermal expansion of the materials.

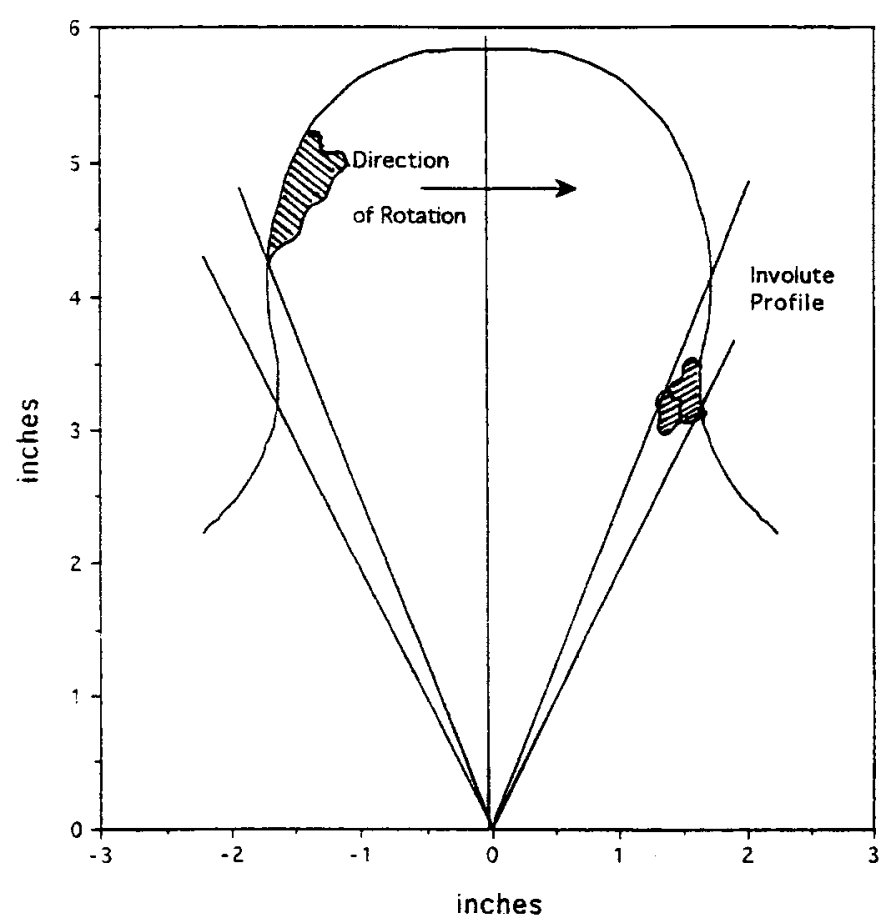

FIGURE 1-2
Prior to the present study, no quantitative stress and displacement analysis had been performed for the MultiRecompression Heater. Because the failure was caused by severe impeller contact, analysis of the displacement of the impeller surface is especially important. The present research considers how the displacement field within the impeller depends on various thermal and mechanical forces, geometric configurations, and material composition. Such research is possible at this time because of advances in scientific computers and development of sophisticated finite element techniques.

\section{PROBLEM FORMULATION}

\subsection{Basic Assumptions}

Thermal, centrifugal and pressure loads will be considered as the main loads affecting the stress and displacement distributions. The properties of the material, the rotational speed and the geometry are the main additional factors influencing these distributions. Analysis will be focused on characteristics of the temperature distribution within the impeller, the maximum surface displacement, and the greatest stress in the impeller.

Figure 1-3 is a sketch of a representative MultiRecompression Heater employing four-lobe rotors. The surface of each rotor is composed of eight circular arcs of the same radius which are tangent at their points of coincidence. The temperature difference between the flow inlet and outlet produces a periodic thermal boundary condition on the impeller surface. The resulting non-uniform temperature distribution causes thermal stresses within the impellers. The pressure difference between inlet and outlet causes a distributed normal load applied to the surface of the impeller. And, because of the high-speed rotation, a centrifugal load acts as a body force in the stress analysis.

The gas flow field in the Multi-Recompression Heater influences the stress analysis through the heat transfer to 


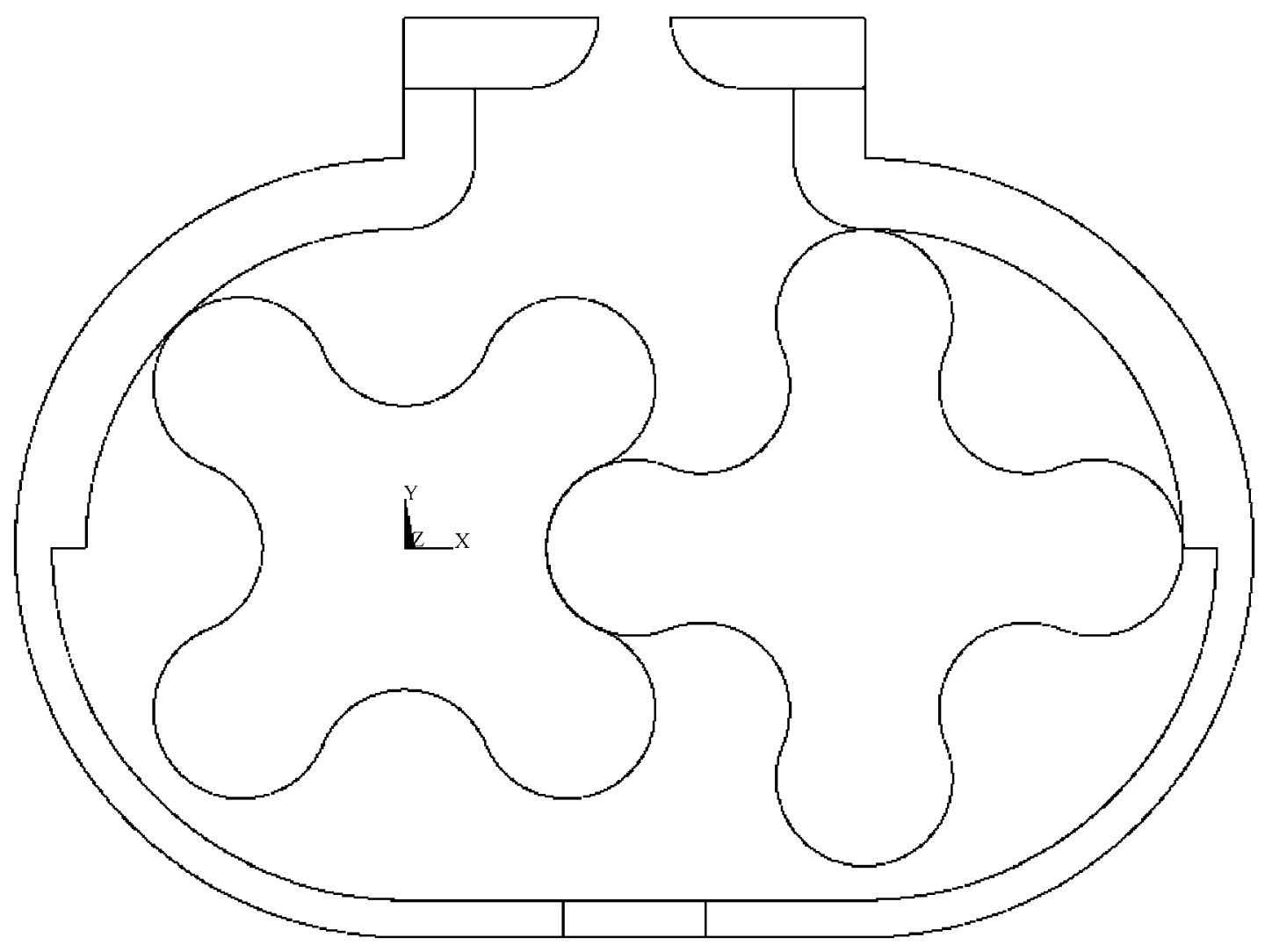

FIGURE 1-3

the impeller. In this study, the flow and temperature fields in the gas are not evaluated. Rather, their influence on the heat transfer and temperature field in the impeller is implicitly included through estimates made for the convective heat transfer coefficients and the variation of the bulk gas temperature seen by the impeller during its revolution.

The idealized mathematical model of the impeller lobes is based on the following assumptions:

- The load on the impeller is not a function of axial position ( $z$ direction).

- The deformations are small.

- The materials composing the impeller are isotropic.

- The material behaves elastically or nonlinear elastically at all times. Nonlinear elasticity is due to temperature dependent elastic properties.

- The thermal field is two-dimensional; i.e., there is of no heat loss in the axial direction $(z)$.

\subsection{Governing Equations}

The thermal energy equation is given by:

$$
\nabla \cdot(K \nabla T)+\dot{q}=\rho c \frac{\partial T}{\partial t}
$$

where $T$ is temperature, $\rho$ is density, $c$ is specific heat, $K$ is thermal conductivity, and $t$ is time. The term $\dot{q}$ is a distributed heat source/sink due to, say, internal cooling of the impeller. The thermal conductivity and the specific heat are taken to be temperature dependent. When internal cooling is not used and direct thermo-mechanical coupling is small (the usual case), then the heat source term $\dot{q}$ equals zero and Eq. [2-1] reduces to:

$$
\nabla \cdot(K \nabla T)=\rho c \frac{\partial T}{\partial t}
$$

The equations of mechanical equilibrium are:

$$
\begin{gathered}
\frac{\partial \sigma_{x x}}{\partial x}+\frac{\partial \sigma_{x y}}{\partial y}+\frac{\partial \sigma_{x z}}{\partial z}+F_{x}=0 \\
\frac{\partial \sigma_{x y}}{\partial x}+\frac{\partial \sigma_{y y}}{\partial y}+\frac{\partial \sigma_{y z}}{\partial z}+F_{y}=0 \\
\frac{\partial \sigma_{x z}}{\partial x}+\frac{\partial \sigma_{y z}}{\partial y}+\frac{\partial \sigma_{z z}}{\partial z}+F_{z}=0
\end{gathered}
$$

The strain-displacement relations are:

$$
\begin{aligned}
\varepsilon_{x x} & =\frac{\partial u}{\partial x} \\
\varepsilon_{y y} & =\frac{\partial v}{\partial y} \\
\varepsilon_{z z} & =\frac{\partial w}{\partial z}
\end{aligned}
$$




$$
\begin{aligned}
& \varepsilon_{x y}=\frac{1}{2} \gamma_{x y}=\frac{1}{2}\left(\frac{\partial u}{\partial y}+\frac{\partial v}{\partial x}\right) \\
& \varepsilon_{y z}=\frac{1}{2} \gamma_{y z}=\frac{1}{2}\left(\frac{\partial v}{\partial z}+\frac{\partial w}{\partial y}\right) \\
& \varepsilon_{z x}=\frac{1}{2} \gamma_{z x}=\frac{1}{2}\left(\frac{\partial w}{\partial x}+\frac{\partial u}{\partial z}\right)
\end{aligned}
$$

where $u, v, w$ are the displacement components in the $x, y, z$ directions; $\varepsilon_{x x}, \varepsilon_{y y}, \varepsilon_{z z}$ are the normal strains $\gamma_{x y}, \gamma_{y z}, \gamma_{z x}$ are the shear strains; $\sigma_{x x}, \sigma_{y y}, \sigma_{z z}$ are the normal stresses; and $\sigma_{x y}, \sigma_{x z}, \sigma_{y z}$ are the shear stresses.

The constitutive relations are given by:

$$
\begin{aligned}
\varepsilon_{x x} & =\frac{1}{E}\left[\sigma_{x x}-\nu\left(\sigma_{y y}+\sigma_{z z}\right)\right]+\alpha \cdot\left(T-T_{a}\right) \\
\varepsilon_{y y} & =\frac{1}{E}\left[\sigma_{y y}-\nu\left(\sigma_{z z}+\sigma_{x x}\right)\right]+\alpha \cdot\left(T-T_{a}\right) \\
\varepsilon_{z z} & =\frac{1}{E}\left[\sigma_{z z}-\nu\left(\sigma_{x x}+\sigma_{y y}\right)\right]+\alpha \cdot\left(T-T_{a}\right) \\
\varepsilon_{x y} & =\frac{1}{2 G} \sigma_{x y} \\
\varepsilon_{y z} & =\frac{1}{2 G} \sigma_{y z} \\
\varepsilon_{z x} & =\frac{1}{2 G} \sigma_{z x}
\end{aligned}
$$

where $\alpha$ is the coefficient of thermal expansion, $T_{a}$ is the temperature at which the MRH is assembled, $E$ is the elastic modulus, $\nu$ is Poisson's ratio, and $G$ is the shear modulus. Note that $E, G$ and $\nu$ are related by:

$$
G=\frac{E}{2(1+\nu)}
$$

\subsection{Boundary Conditions}

For the thermal field, the initial condition is

$$
T(\vec{r}, 0)=T_{a}
$$

The convective boundary condition is given by

$$
-K\left(\frac{\partial T}{\partial n}\right)_{S}=h\left(T-T_{f}(t)\right)
$$

where $n$ represents the outward normal to the surface $S$, and $h$ is the convective heat transfer coefficient (which is a function of the flow field). $T_{f}(t)$ is the temperature of the bulk gas seen by a given point on the impeller; it varies periodically, and the high temperature portion increases with rotational speed.

For the stress field, the general types of boundary conditions are: surface force, surface displacement, and combinations of both. In the present work, the section of the impeller boundary which interacts with the flow field experiences surface force boundary conditions. Generally, normal stresses (pressure) are prescribed; shear stresses are negligible. The boundary of the impeller connected to the axis is always assigned zero displacement in the radial direction.

\subsection{Material Properties}

Usually the behavior of real materials is highly complex and is influenced by such factors as the thermodynamic state (temperature, pressure, humidity, etc.), the magnitude and nature of the loads, the time, the rate of loading, and the previous history of the material (Bathe, 1996). Providing a means for evaluating materials is an important of the present study.

The significant advantage of the MRH is its ability to supply clean, uniformly heated, very high temperature gases. During a single revolution, the impellers are therefore subjected to both a high temperature environment and a large gas temperature variation. Therefore, the thermal and mechanical properties $(K, \alpha, E)$ of the materials are considered to be functions of temperature. Their temperature dependence affects the development of thermal stresses in the impellers and the magnitudes of surface displacements.

\section{METHODOLOGY}

As discussed in the previous section, even an idealized MRH model is characterized by a complicated geometry, periodic boundary conditions and non-linear governing equations (arising from temperature dependent material properties). This makes it impossible to obtain an analytical solution. In this section, a model for the MRH impeller will first be established. Then, alternative approaches to solving the problem will be discussed. Finally, numerical solutions will be presented.

\subsection{Numerical Technique}

The ABAQUS finite element code was used to obtain numerical solutions. The analysis was carried out for 2-D and 3-D models. The respective finite element meshes are shown in Figure 3-1. An automesh program (Song \& Wang, 1999) was used to generate them.

\subsection{Boundary Conditions}

Based on the discussion in Section 2, it is seen that the problem exhibits several characteristics:

- The geometric domain is symmetric; the boundary conditions are not. 

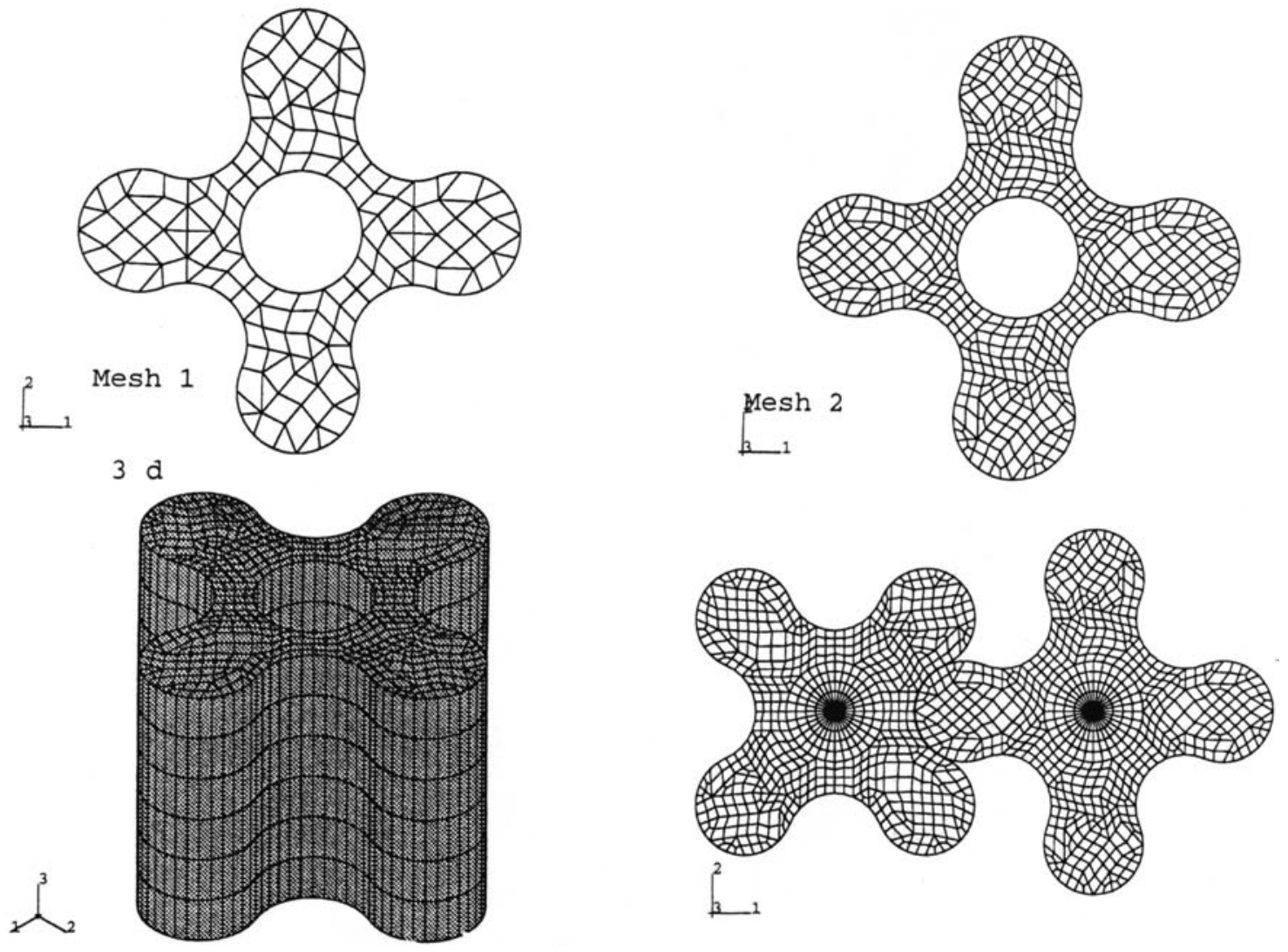

FIGURE 3-1

- The material properties introduce nonlinearity due to their dependence on temperature.

- The boundary condition for the temperature distribution is periodic but not axisymmetric.

- The pressure load is generally not axisymmetric. It is, however, independent of the axial direction $(z)$.

- The centrifugal load is a function of the rotational speed, material density, and size of the MRH.

A steady-periodic convective boundary condition is applied in the thermal field analysis. It depends on the compressed gas temperature, the rotational speed, and the convective heat transfer coefficient. In the actual device, when the impeller turns at a certain RPM the gas temperatures and pressures become essentially fixed in the upper and lower plenums of the MRH. Higher RPM produces higher outlet plenum temperatures and pressures. In the finite element model, the coordinate system is fixed to the impeller and the gas temperature which a point on its surface sees during one revolution is modeled as periodically changing with time.

To simplify application of the convective boundary condition, the local gas temperature is represented by an $\mathrm{N}$-term Fourier series and applied simultaneously to each of four sections of the impeller boundary $(i=1,2,3,4)$ :

$$
\begin{aligned}
T_{f i}(t)= & A_{0}+\sum_{n=1}^{N}\left[A_{n} \cos \left(n \omega\left(t-t_{0 i}\right)\right)\right. \\
& \left.+B_{n} \sin \left(n \omega\left(t-t_{0 i}\right)\right)\right]
\end{aligned}
$$

where $A_{0}, A_{n}, B_{n}$, and $t_{0 i}$ are functions of the rotational speed, compressed gas temperature, and inlet temperature. The time shift, $t_{0 i}$, between each section of the surface depends on the rotational speed and the number of boundary regions. For the present four-region model, the time shift is equivalent to one-fourth of a revolution. For a rotational speed of $1000 \mathrm{RPM}, t_{01}, t_{02}, t_{03}$, and $t_{04}$ are equal to $-0.03,-0.015,0$, and 0.015 seconds, respectively. The convective heat transfer coefficient depends on the flow and temperature fields. In the present study, it is calculated from empirical relations. $\left(\mathrm{Nu}=h l / k=C \operatorname{Re}^{n} \operatorname{Pr}^{m}\right.$, where $\mathrm{Re}$ is the Reynolds number, $\operatorname{Pr}$ is the Prandtl number.)

The pressure variation around the surface of the impeller produces a steady-periodic pressure load which depends on the rotational speed. Similar to the thermal boundary condition, the pressure load is applied to four parts of the 
boundary separately, each part represented by a finite Fourier series; i.e.,

$$
\begin{aligned}
P_{i}(t)=C_{0}+\sum_{n=1}^{N} & {\left[C_{n} \cos \left(n \omega\left(t-t_{0 i}\right)\right)\right.} \\
& \left.+D_{n} \sin \left(n \omega\left(t-t_{0 i}\right)\right)\right]
\end{aligned}
$$

where $C_{0}, C_{n}, D_{n}$, and $t_{0 i}$ are functions of the rotational speed, the compressed gas and inlet pressures. As with the thermal field, the time delays are one-fourth of a revolution between each $P_{i}(t)$.

As noted earlier there are two general types of boundary conditions for the stress field analyses: force and displacement. When displacement boundary conditions are applied, it is necessary to use additional zero displacement conditions in order to eliminate artificial rigid body motion.

\subsection{Stress Model}

For the thermal analysis, a 2-D mechanical model was chosen because of its symmetrical geometry and simpler boundary conditions. For the stress analysis, the 3-D model is more accurate; however, the computer time and storage increase substantially.

There are two types of 2-D models: plane strain and plane stress. Plane strain is used in analyzing a long body whose geometry and load do not vary in the longitudinal direction, and all applied loads are perpendicular to the longitudinal direction. Usually it is necessary that the length of the body be large compared to the maximum cross sectional dimension.

In contrast, the plane stress condition is characterized by a longitudinal dimension which is much smaller than the $x$ and $y$ dimensions. Also, its geometry and load do not vary longitudinally, and all applied loads are perpendicular to the longitudinal direction.

It is to be noted that thermal stress is developed by differential volumetric (3-D) expansion or contraction. If artificially constrained in one direction, artificial stresses will be generated. It is therefore anticipated that plane stress will be a better 2-D model than plane strain for calculating thermal stresses.

\subsection{Material Properties}

The original MRH was constructed of steel, but aluminum and graphite are also considered in the present calculations. Aluminum is a common material for superchargers and was used to build a laboratory prototype of the MRH for experiments related to this project. Graphite is a material more appropriate for the very high temperature environment in which the MRH is intended to operate. Comparisons will be made among these materials for various

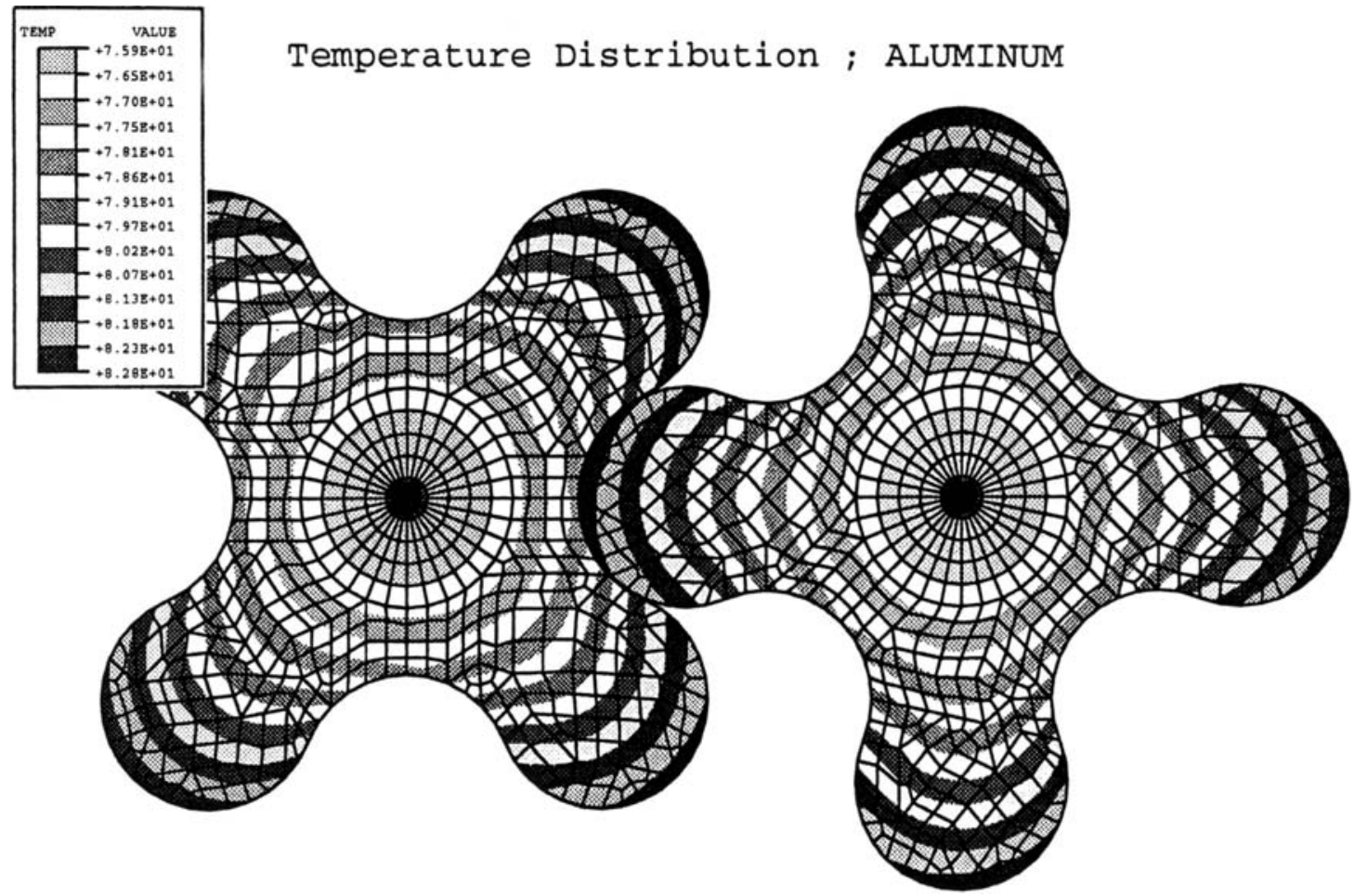

FIGURE 4-1 


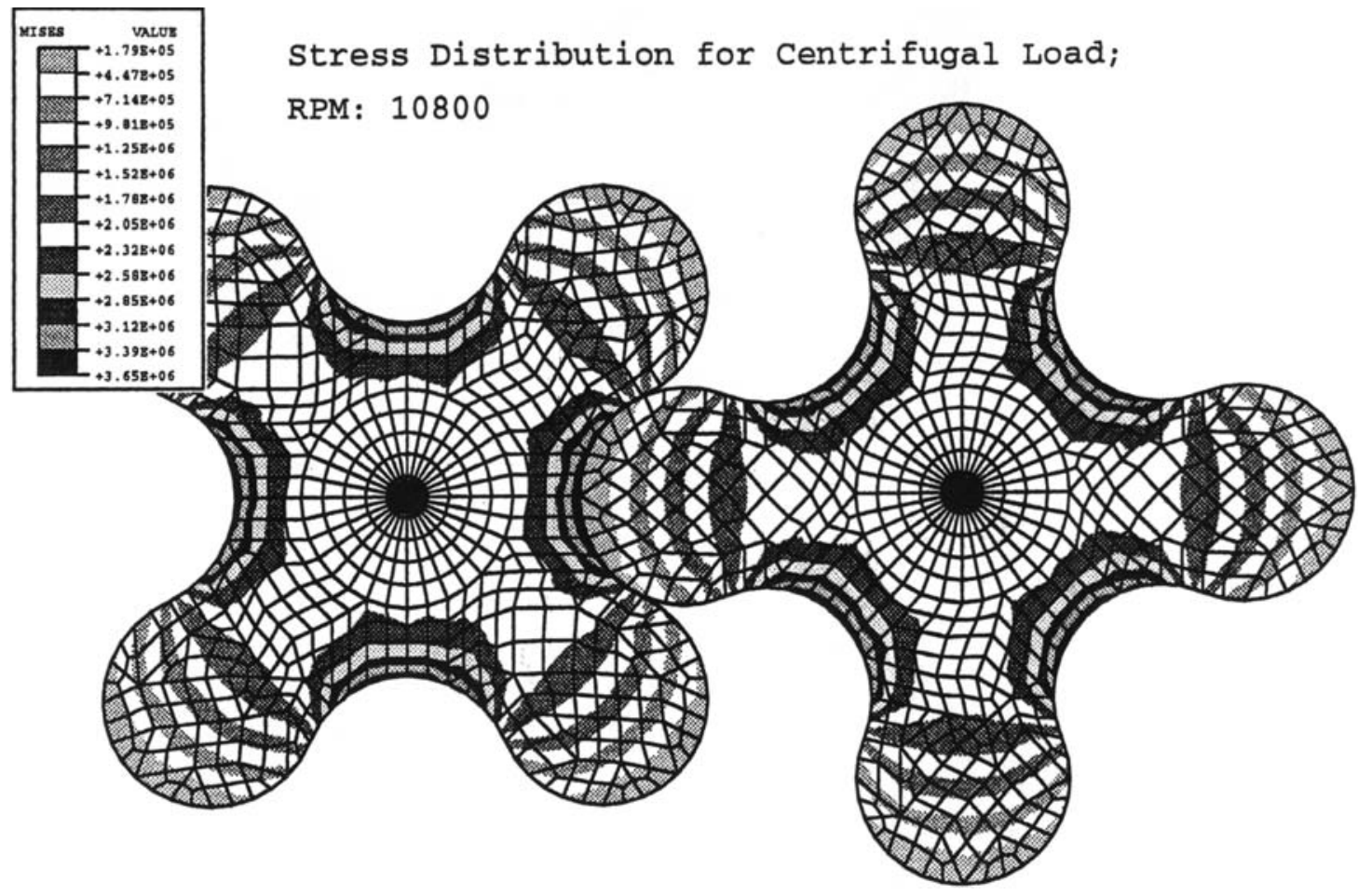

FIGURE 4-2

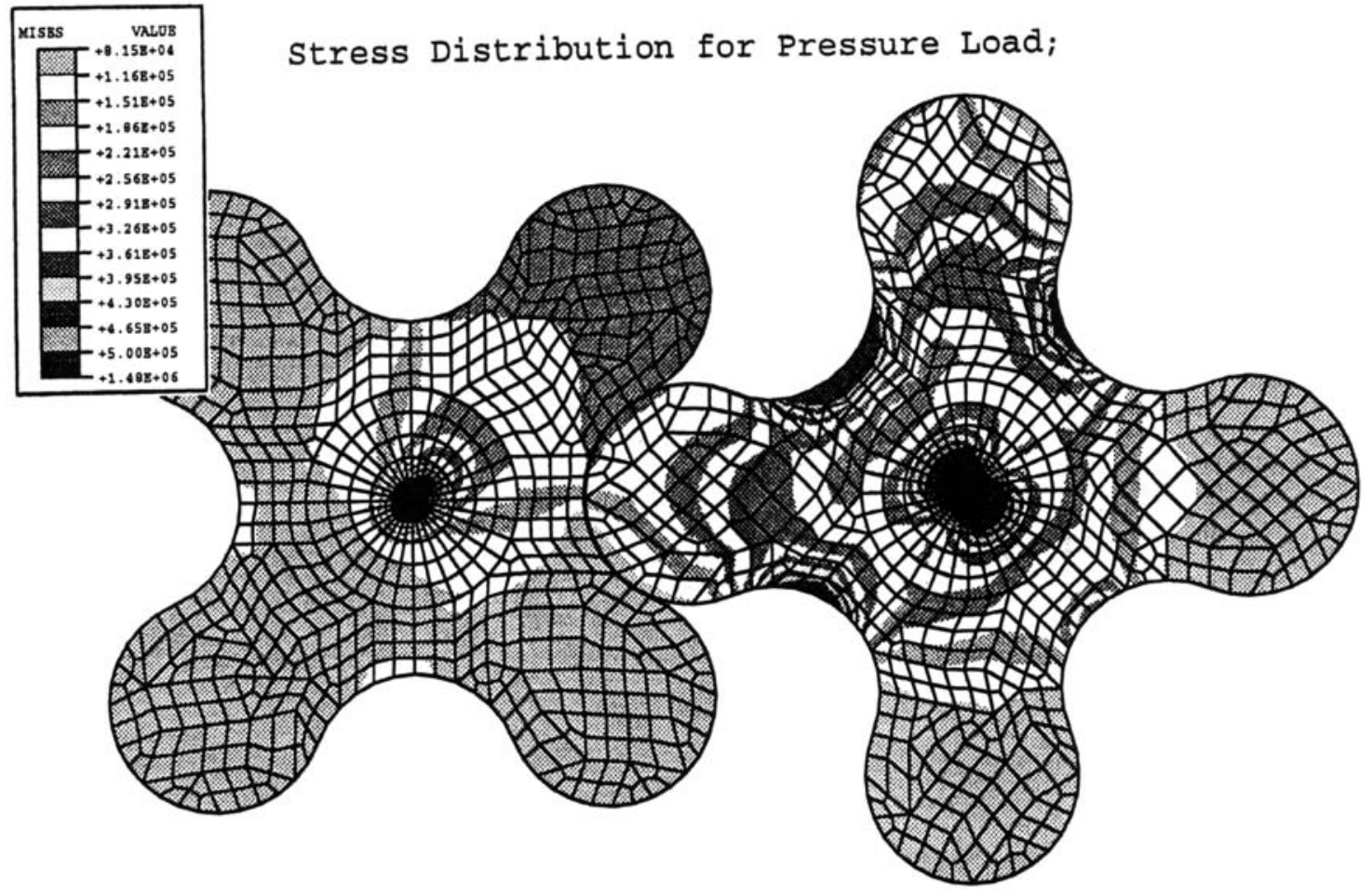

FIGURE 4-3 
loadings and boundary conditions. The properties of these materials at room temperature and their variations with temperature are detailed in the references (Davis, 1993; Davis, 1998; Brixius, 1987 and UCAR Carbon Company, 1991).

\subsection{Coupled Versus Uncoupled Analysis}

Two different computational approaches are tested. The first is a coupled analysis where the thermal and mechanical solutions are obtained simultaneously with the stress and thermal analyses being interdependent.

The second approach is an uncoupled analysis in which the temperature distribution is computed independently from the mechanical deformation, and then used as input to the stress analysis. This is a reasonable approach if the coupling between the stress field and the temperature distribution is weak. The uncoupled approach saves a lot of computational time compared to the coupled analysis in which all fields need to be computed simultaneously.

The results show that the difference between the two approaches is very small. Therefore, the uncoupled analysis was mainly used in the present work.

\section{RESULTS}

Since the uncoupled thermal-stress analysis is mainly used to solve the problem, the stress analysis for the impeller uses as inputs the results of a thermal analysis combined with imposed pressure and centrifugal loads. It is important to note that superposition of the results due to each load can not be done here to obtain the combined effect because of the nonlinear characteristics of the problem. However, example calculations based on the hypothetical imposition of each type of loading separately are made for special comparison purposes only.

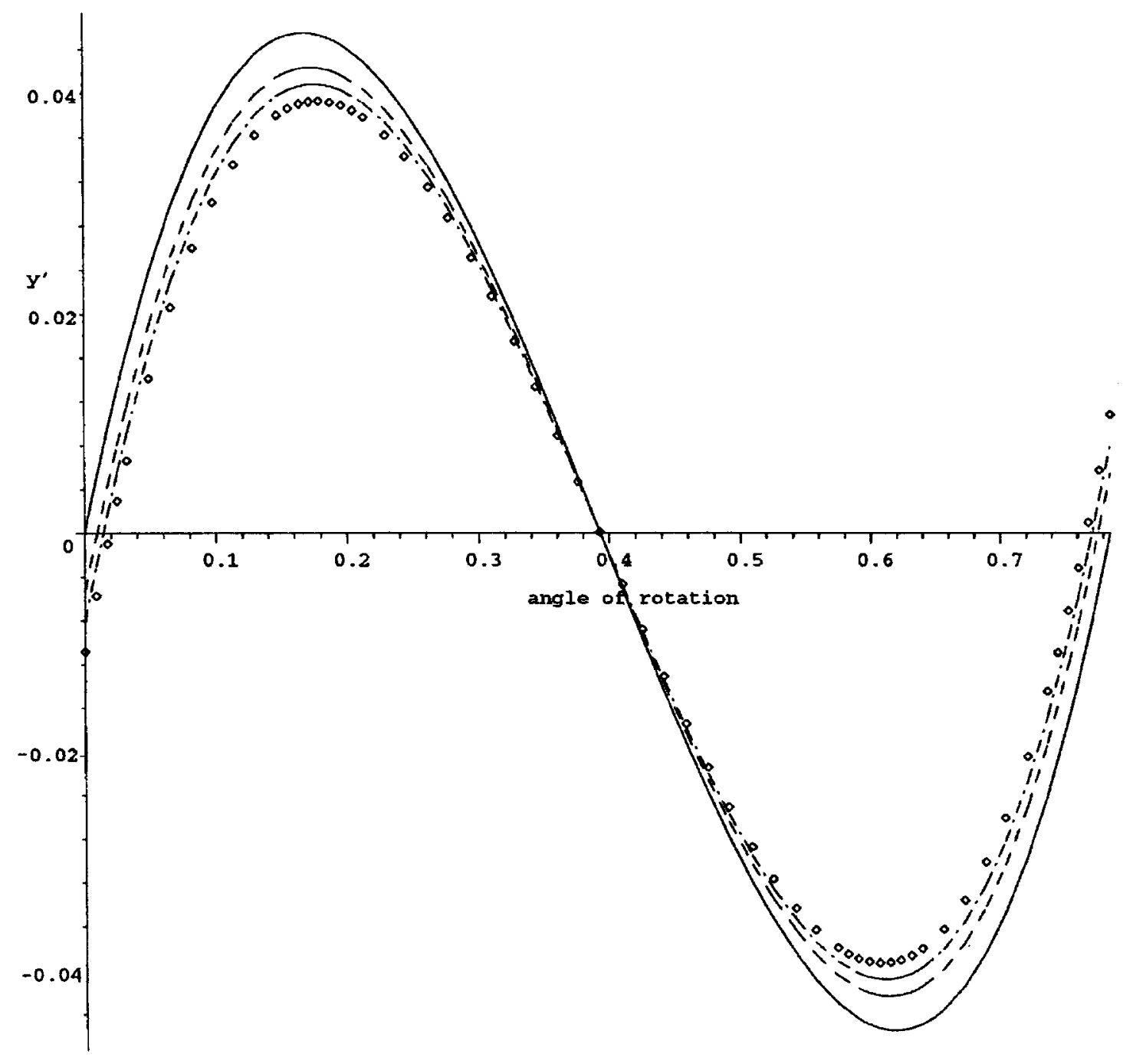

FIGURE 4-4 
Of particular interest in numerical calculations are the following:

\subsection{The Temperature Field in the Impeller}

The temperature field is the input data for the thermal stress analysis. From the computations, several characteristics of the temperature distribution can be noted:

- The temperature gradients in the impeller are small. This is because the Biot number $(h R / k)$ is small, where $R$ is the overall impeller radius;

- The temperature distribution within the impeller lobes is almost axisymmetric even though the transient convective boundary condition is not (see Figure 4-1). This is because the rotational speed is high and the Biot number is small, thereby reducing the influence of non-axisymmetric conditions on the boundary;

- The temperature level of the impeller lobes depends mainly on the compressed gas and inlet gas temperatures. In the $\mathrm{MRH}$, the lobes pass alternately through high and low temperature gases with only a short residence time in each. They also pass through two other regions: one facing the casing and one facing the other impeller. Consequently, when steady-periodic behavior is achieved, the temperature level in the impeller becomes (as a result of the small Biot number) approximately a weighted average of the compressed and inlet gas temperatures;

- The choice of materials is important. The material which has the lower volumetric heat capacity (product of density and specific heat) will reach the steady-periodic state faster.

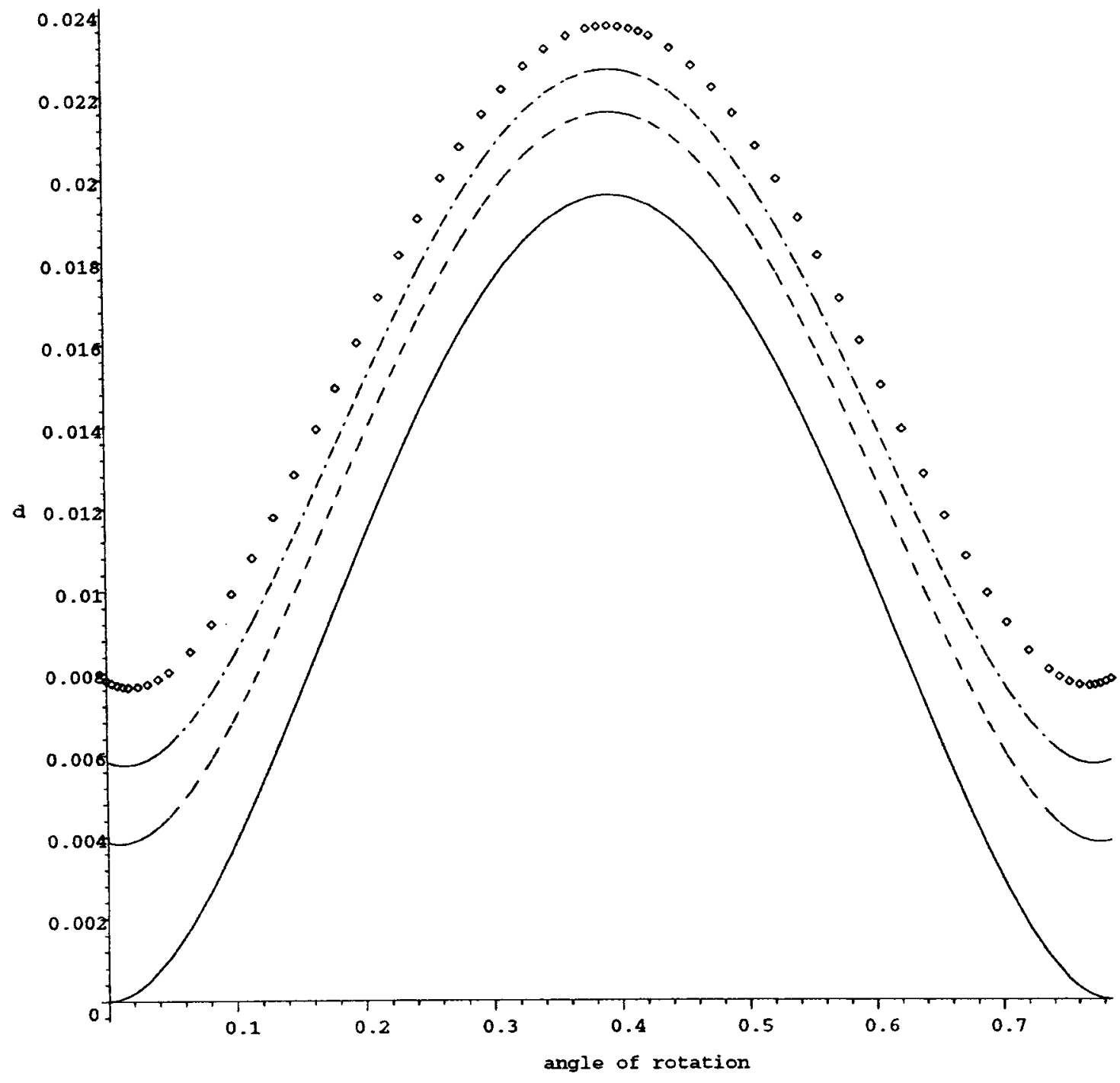

FIGURE 4-5 


\subsection{Stress Distribution in the Impeller}

From the results of the thermal analysis, the temperature gradients in the impeller are not large. Therefore, the thermal stress is small even though the displacement due to the nearly uniform thermal expansion is large.

The centrifugal load is a function of the geometry, materials and rotational speed. As shown in Figure 4-2, the stress distribution is axisymmetric, and the stress is largest in the well region.

The pressure load is periodic with a frequency proportional to the rotational speed. However, it is not axisymmetric and thus the stress distribution is not axisymmetric (see Figure 4-3).

The non-linear analysis (temperature dependent properties) shows the stress level to be $10-20$ percent lower than that predicted by the linear analysis. However, since the yield strength of aluminum decreases almost 50 percent from room temperature to $205^{\circ} \mathrm{C}$, temperature dependent properties must always be considered in the stress analysis for the impeller of the MRH.

\subsection{Surface Displacement and Minimum Clearance}

The failure of the MRH at LLNL was caused by severe impeller contact. The clearance between the two impellers is a very important parameter. Increasing the clearance decreases the efficiency. Establishing criteria to define the cold (assembly) clearance in a new design is essential. The analysis shows that the minimum clearance in the MRH is a function of the largest surface displacement, the initial clearance at assembly (the cold clearance), and the angular orientation of the impeller. Equations were derived which allow this quantity to be calculated (Wang, 1999).

The minimum clearance under operating conditions equals the cold minimum clearance minus the displacements of the two meshing lobes. Figure 4-4 shows how the angle of rotation at which the minimum clearance occurs depends on the initial clearance. The abscissa is the rotation angle $\eta$. The ordinate is the derivative of the cold minimum clearance with respect to the angle of rotation, $d \delta_{c, \min } / d \eta$. The minimum clearance occurs at the angle of rotation where this derivative equals zero. The variation of

Material: Graphite,Thermal load: Th $800 \mathrm{C}, \mathrm{Tl} 80 \mathrm{C}, \mathrm{h} 200 \mathrm{w} / \mathrm{m}^{\star} \mathrm{m}$ C
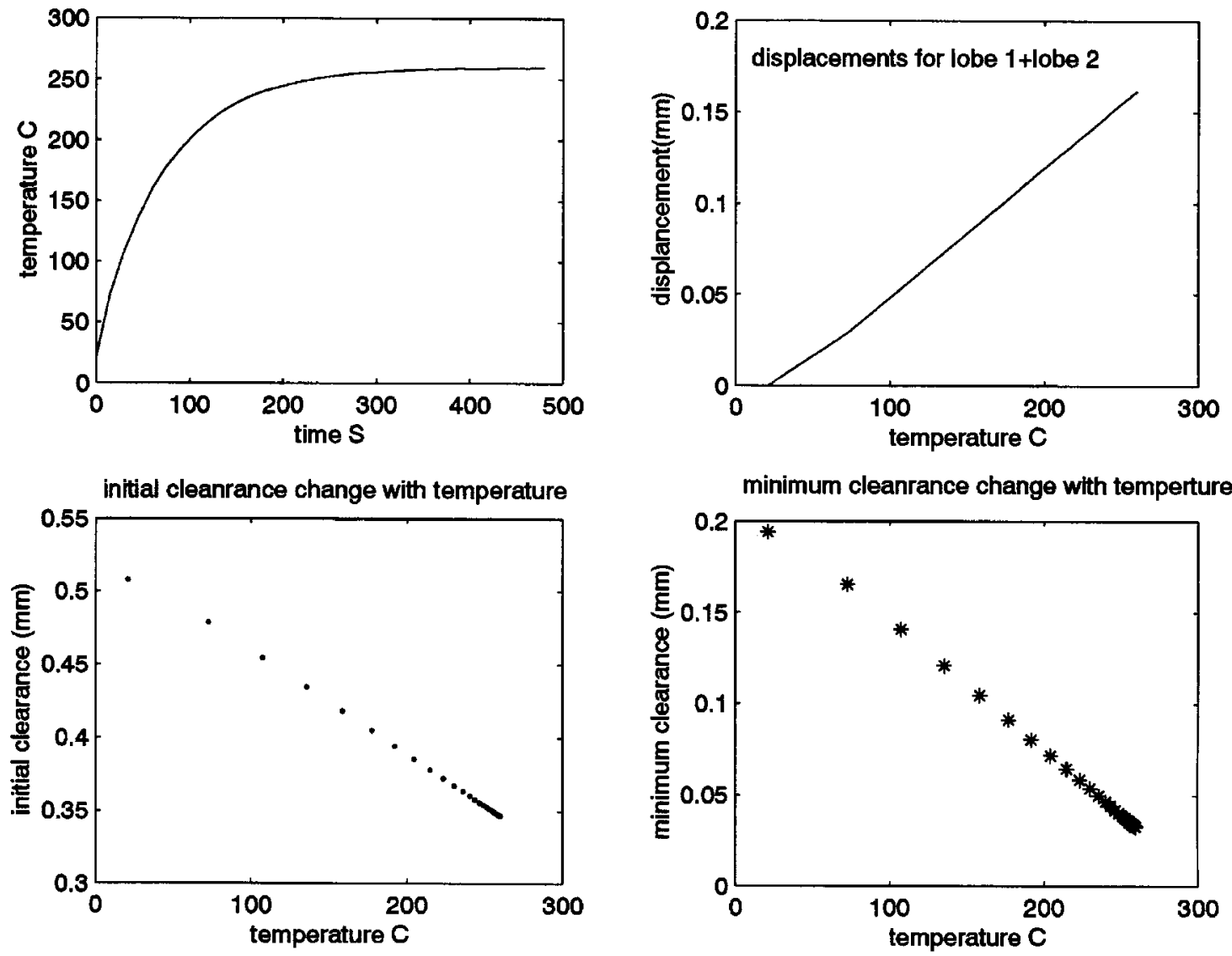

FIGURE 4-6 
TABLE I Natural frequency for different materials

\begin{tabular}{lcccc}
\hline & & Steel & Aluminum & Graphite \\
\hline Density & $\mathrm{kg} / \mathrm{m}^{3}$ & 8680 & 2685 & 1780 \\
Modulus & $\mathrm{N} / \mathrm{m}^{2}$ & $210 * 10^{9}$ & $72 * 10^{9}$ & $12 * 10^{9}$ \\
Mode 1 & $\mathrm{Hz}$ & 8680 & 8779 & 4774 \\
Mode 2 & $\mathrm{Hz}$ & 13069 & 13179 & 7188 \\
Mode 3 & $\mathrm{Hz}$ & 14537 & 14705 & 7996 \\
Mode 4 & $\mathrm{Hz}$ & 14540 & 14707 & 7997 \\
Mode 5 & $\mathrm{Hz}$ & 26390 & 26530 & 14516 \\
\hline
\end{tabular}

TABLE II Natural Frequency for different models (aluminum)

\begin{tabular}{lccc}
\hline Mode no. & $3 \mathrm{D}$ & $\begin{array}{c}\text { Plane strain } \\
\mathrm{Hz}\end{array}$ & $\begin{array}{c}\text { Plane stress } \\
\mathrm{Hz}\end{array}$ \\
\hline 1 & $\mathrm{~Hz}$ & 8872 & 8620 \\
2 & 8800 & 13389 & 12966 \\
3 & 8873 & 14820 & 14151 \\
4 & 13283 & 14840 & 14161 \\
5 & 13401 & 26643 & 25631 \\
6 & 14340 & 29177 & 26864 \\
\hline
\end{tabular}

the cold minimum clearance with angle of rotation and initial clearance is shown in Figure 4-5.

The lobe displacements depend on the thermal and mechanical loads, and the material properties. For the same thermal load, the displacements in the lobes made from graphite are about one-third of those made from aluminum. The displacement for aluminum is $7 \%$ greater when computed for temperature varying properties rather than for constant, room temperature properties (under the same loading). It is also to be noted that the minimum clearance changes during the transient heat-up process as shown in Figure 4-6.

\subsection{Natural Frequency Analysis}

This analysis yields the natural frequencies and corresponding mode shapes of the system. It helps in deciding whether a dynamical analysis is needed. Dynamic analysis is required under certain loading conditions. If the load is cyclic but with a frequency less than about one-third of the structure's lowest natural frequency, the problem can be considered quasistatic. If the load has a higher frequency,

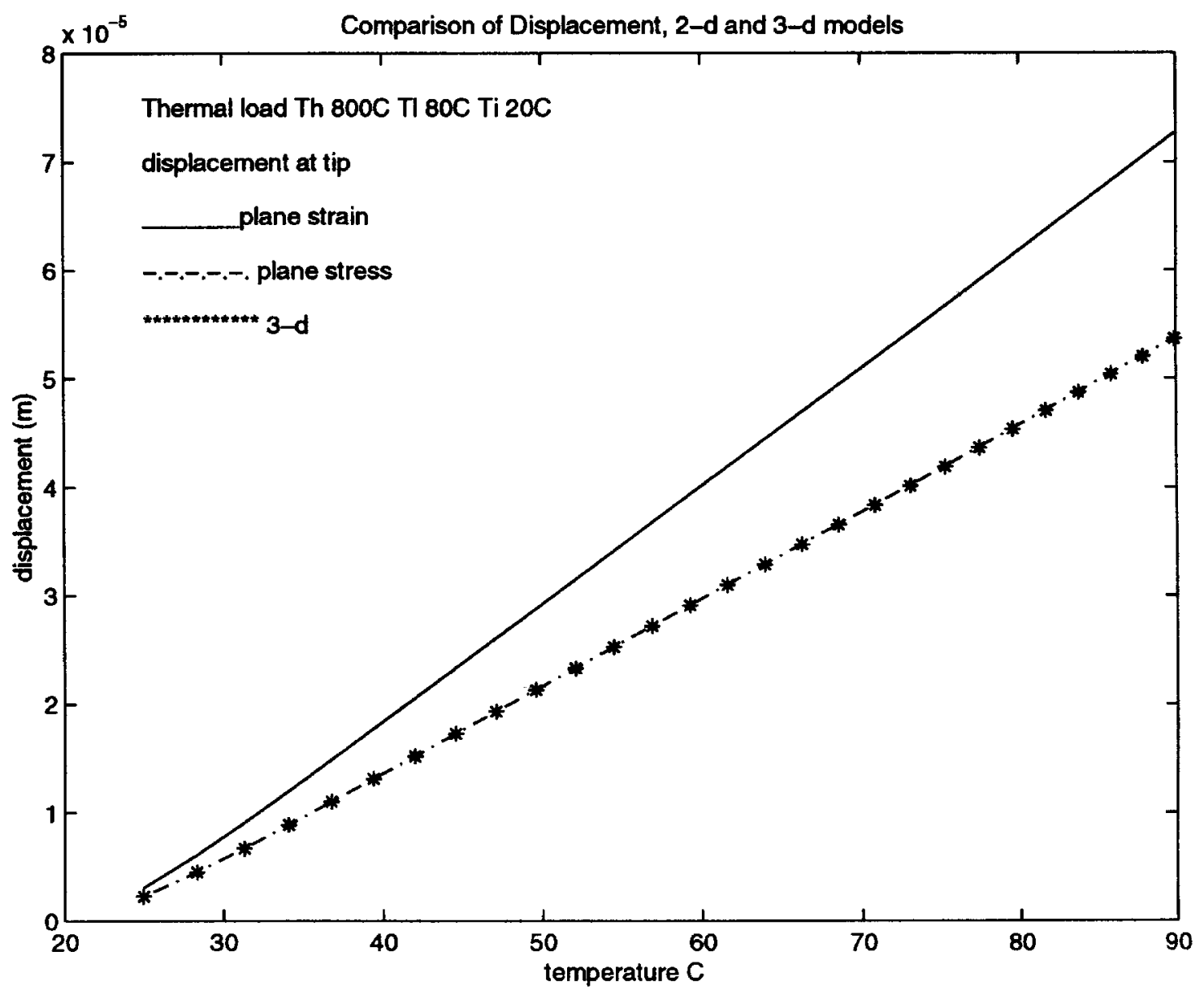

FIGURE 4-7 
varies randomly, or is applied suddenly, then a dynamic analysis is required (Cook, 1995). For rotating machinery, the possibility of resonance also needs to be considered.

For the MRH, the pressure load on an impeller is periodic with a frequency proportional to the rotational speed. For RPM ranging from 1000 to 10800 , the frequency of the pressure load varies from 16.7 to $180 \mathrm{~Hz}$.

The results from the natural frequency analysis of the MRH using the plane strain model are summarized in Table I for three different materials. Table II shows results for aluminum using different analysis models. The lowest six vibrational modes are listed in the tables. The results indicate that resonance will not occur within the range of the rotational speed of interest. Therefore, a dynamic analysis is not required to characterize the MRH operating behavior.

\subsection{Comparison of 2-D and 3-D Stress Analysis}

Comparison cases for 2-D and 3-D analyses for purely centrifugal loading show that the plane stress model is closer to the 3-D results than the plane strain model. The difference between the 2-D and 3-D results is not large. For a purely thermal load, the plane stress model is quite accurate. The plane strain model, however, gives much higher stresses than the 3-D model. This is due to the unrealistic assumption of no thermal strain in the $z$-direction, which generates artificial $z$-direction normal stresses.

Since thermal expansion is a major factor in the analysis, the plane strain model is not useful. By contrast, Figure 4-7 demonstrates that the displacements predicted by the 2-D plane stress model are very close to those of the 3-D model. In summary, it is seen that for the present study the 2-D plane stress model is an accurate approximation to the 3-D stress/displacement behavior and can be used as a computationally efficient approximation to the full 3-D calculations.

\section{CONCLUSIONS}

The main conclusions that can be drawn from the present work are as follows. First, a model was developed which can be used not only to gain detailed understanding of the MRH impellers, but also to provide the design parameters for new impellers. Second, results from the thermal analysis indicate that the temperature variation throughout the impeller is small for the geometry, materials and transient boundary conditions of interest. The temperature level in the impeller is approximately a weighted average of the compressed gas and inlet gas temperatures. Therefore, internal cooling of the impeller may be avoided if its material is chosen properly for different compressed gas temperatures. Third, stress analysis shows that the von
Mises stress under combined thermal, centrifugal, and pressure loads is less than the yield stress. Finally, the computations indicate that POCO graphite is an excellent material for the MRH. Not only can it withstand high temperatures where conventional materials like aluminum or steel would melt, but its thermal and mechanical properties are exceptional: relatively low coefficient of thermal expansion, and a Young's modulus $(E)$ which increases with temperature.

\section{Acknowledgments}

This work was supported in part by the Calspan UB Research Center (CUBRC). Discussions with Professor J. C. Mollendorf and Dr. J. A. Lordi were very helpful. The insight of Professor G. F. Dargush regarding finite element computations is also greatly appreciated.

\section{NOMENCLATURE}

$A_{0}, A_{n} \quad$ Fourier coefficients of Fourier cosine series for $T_{f i}(t)$

$B_{n} \quad$ Fourier coefficients of Fourier sine series for $T_{f i}(t)$

$C_{0}, C_{n} \quad$ Fourier coefficients of Fourier cosine series for $P_{i}(t)$

c $\quad$ specific heat $\left(\mathrm{J} / \mathrm{Kg} \cdot{ }^{\circ} \mathrm{C}\right)$

$D_{n} \quad$ Fourier coefficient of Fourier sine series for $P_{i}(t)$

E elastic modulus (Young's modulus)

$G \quad$ shear modulus

$\mathrm{h} \quad$ convective heat transfer coefficient

$K \quad$ thermal conductivity $\left(\mathrm{W} / \mathrm{m} \cdot{ }^{\circ} \mathrm{C}\right)$

$\mathrm{Nu} \quad$ Nusselt number

$P_{i}(t) \quad$ pressure of the gas in MRH

$P_{r} \quad$ Prandtl number

$\dot{q} \quad$ distributed heat source/sink $\left(\mathrm{W} / \mathrm{m}^{3}\right)$

$R_{e} \quad$ Reynolds number

$T \quad$ temperature $\left({ }^{\circ} \mathrm{C}\right)$

$T_{a} \quad$ temperature, at which the MRH is assembled.

$T_{f}(t) \quad$ temperature of the gas in the MRH

$T_{f i}(t) \quad$ temperature of the gas in the region $i$

$t \quad$ time (sec)

$t_{0 i} \quad$ time delay (sec)

$u \quad$ displacement component in $x$ direction

$v \quad$ displacement component in $y$ direction

$w \quad$ displacement component in $z$ direction

\section{Greek Characters}

$\alpha \quad$ thermal expansion $\left(1 /{ }^{\circ} \mathrm{C}\right)$

$\gamma_{x y}, \gamma_{x z}, \gamma_{y z}$ shear strain 


$\begin{array}{ll}\delta_{c, \text { min }} & \text { Clod minimum clearance } \\ \varepsilon_{x x}, \varepsilon_{y y}, \varepsilon_{z z} & \text { normal strain } \\ \eta & \text { angle of rotation of the impeller } \\ \nu & \text { Poisson's ratio } \\ \rho & \text { density }\left(\mathrm{Kg} / \mathrm{m}^{3}\right) \\ \sigma_{x x}, \sigma_{y y}, \sigma_{z z} & \text { normal stresses } \\ \sigma_{x y}, \sigma_{x z}, \sigma_{y z} & \text { shear stresses } \\ \omega & \text { rotation speed }(\mathrm{rad} / \mathrm{sec})\end{array}$

\section{REFERENCES}

Bathe, K. J. (1996) Finite Element Procedures, Prentice Hall, Englewood Cliffs, N. J.

Brixius, W. H. (1987) Properties and Characteristics of Graphite, POCO Graphite, Inc., Decatur, Texas.

Cook, R. D. (1995) Finite Element Modeling for Stress Analysis, Wiley, New York.
Davis, J. R. (1993) ASM Specialty Handbook: Aluminum and Aluminum Alloys, ASM International, Materials Park, $\mathrm{OH}$.

Davis, J. R. (1998) Metals Handbook, Volume 2, Properties and Selection. Nonferrous Alloys and Special-purpose Materials, ASM International, Materials Park, OH.

Dubrin, J. W. and Harri, J. G. (1980) A Mechanical Heater for Production High-temperature Gas Streams, Energy and Technology Review.

Russo, A. L. (1997) A Novel Mechanical Heater for Innovative Pyrolytic Chemical Processes of Current Technological and Environmental Importance.

Song, D. and Wang, H. (1999) Automesh for trimmed surface entity of IGES/PDES, Advances in Engineering Software, 30, 401-406.

UCAR Carbon Company (1991) The Industrial Graphite Engineering Handbook, UCAR Carbon Company Inc., Danbury, CT.

Wang, H. (1999) Thermomechanical Analysis of the Rotating Impeller Lobes of a Roots-type Multi-recompression Heater, Ph.D. Dissertation, Dept. Mech. and Aero. Engineering, State University of New York at Buffalo.

Weatherston, R. C. (1969) The Multi-recompression Heater, A New Concept for Large Scale Hypersonic Testing, AIAA 4th Aerodynamic Testing Conference, Paper No. 69-332, Cincinnati, Ohio/April, $28-30$. 

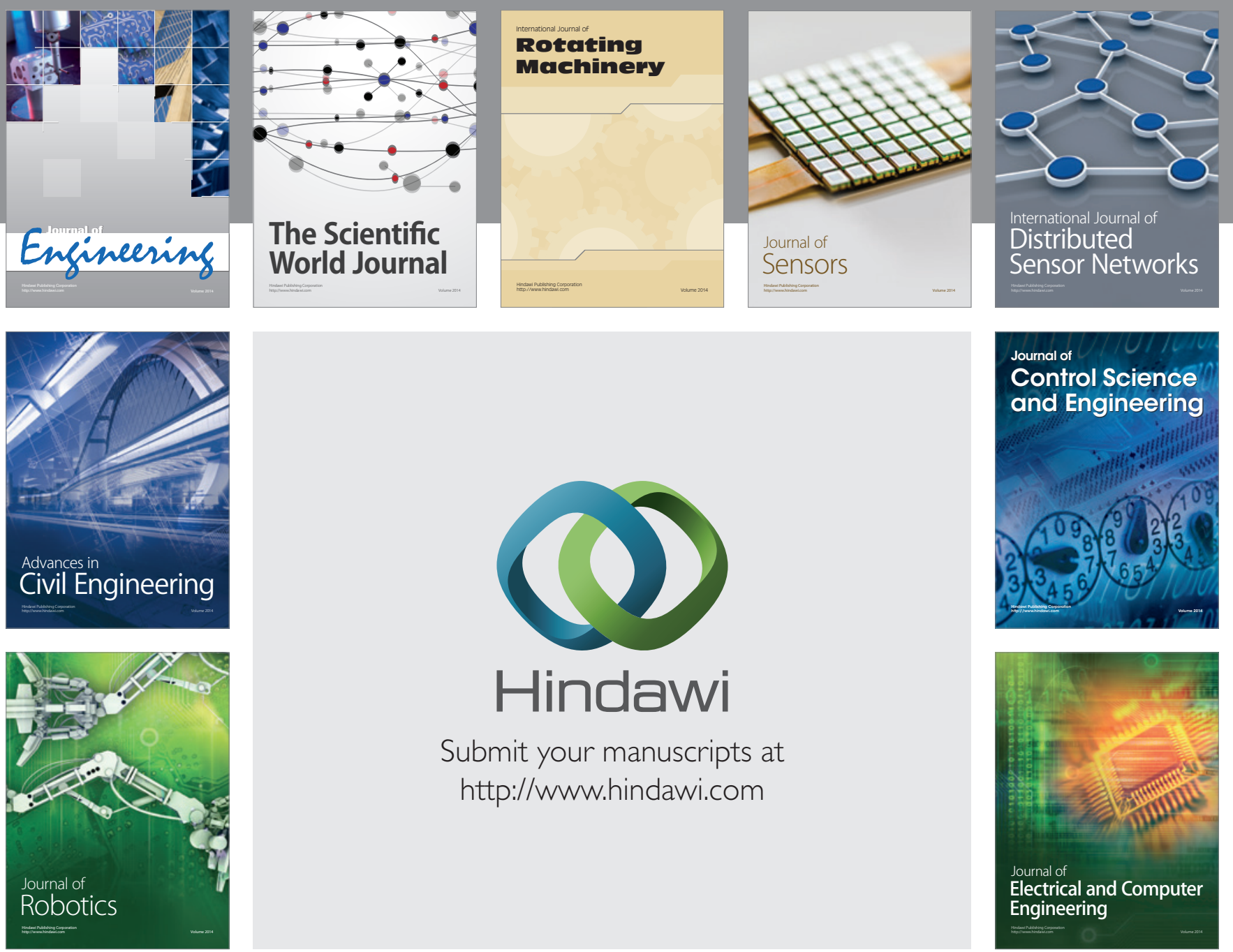

Submit your manuscripts at

http://www.hindawi.com
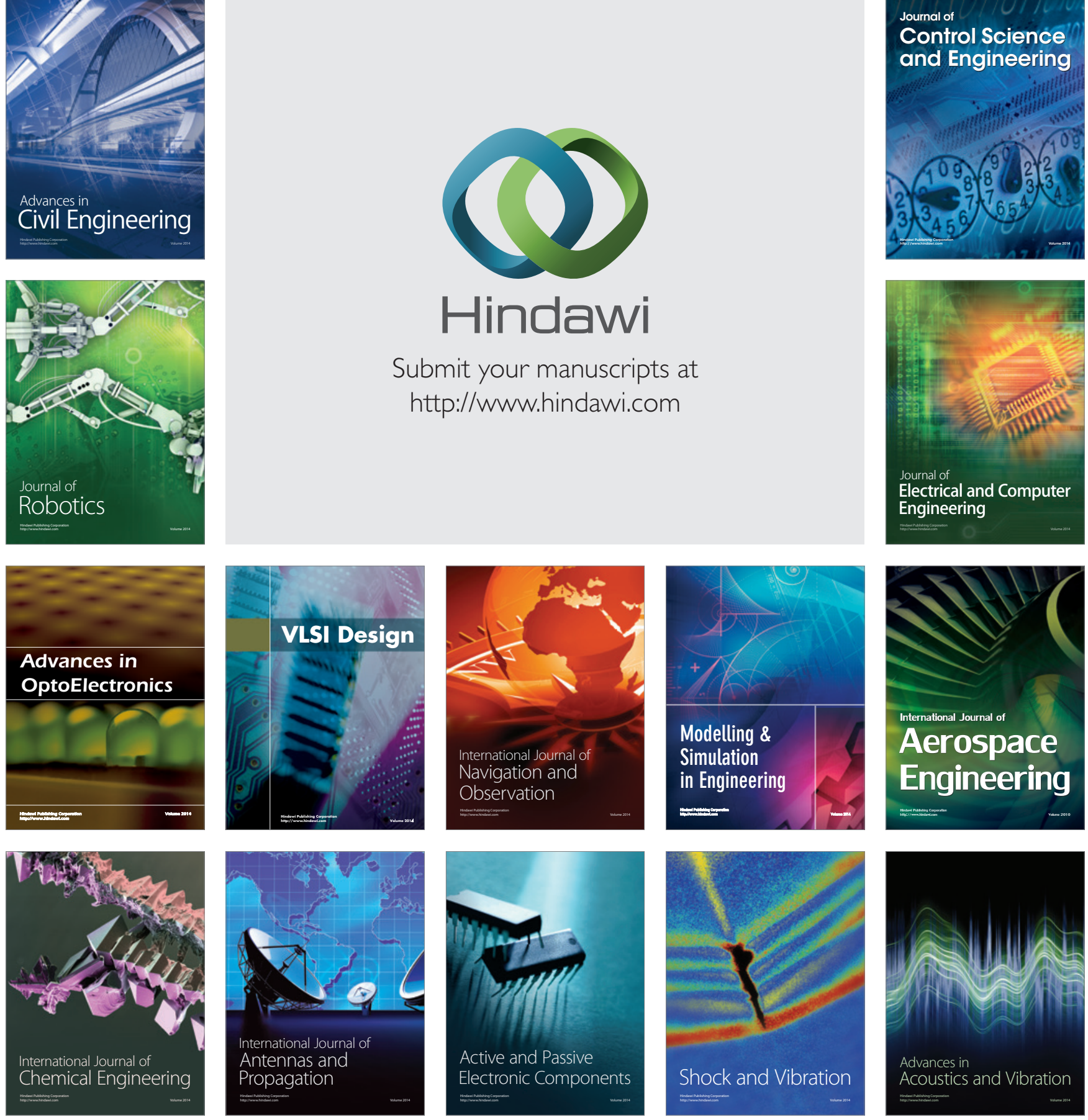